\title{
Advances and Prospects of Frequency Doublers Based On Periodically Poled Silica Fibres
}

\author{
Costantino Corbari $^{1}$, A. Canagasabey ${ }^{1}$, M. Ibsen ${ }^{1}$, A.V. Gladyshev ${ }^{2}$, P.G. Kazansky ${ }^{1}$ \\ ${ }^{I}$ Optoelectronics Research Centre, University of Southampton, Highfield campus, SO17!BJ, Southampton, UK \\ ${ }^{2}$ Fiber Optics Research Center, Russian Academy of Sciences, 38 Vavilov Street, 119333 Moscow, Russia \\ coc@orc.soton.ac.uk
}

\begin{abstract}
Record high efficient frequency doublers in periodically poled silica fibres are demonstrated for light generation at $532 \mathrm{~nm}$ and $775 \mathrm{~nm}$. The onset of nonlinear conductivity is shown to limit the maximum $\chi^{(2)}$ in glass.

OCIS codes: (190.2620) Harmonic generation and mixing; (190.4360) Nonlinear optics, devices; (190.4370)

Nonlinear optics, fibers
\end{abstract}

\section{Introduction}

Periodically poled silica fibres (PPSF) hold promise of circumventing the drawbacks associated with fibre to crystal bulk coupling in second-order nonlinear optical processes. Compared to widely used nonlinear crystals thermally poled silica has a lower second-order nonlinearity $\left(\mathrm{d}_{33} \sim 0.5 \mathrm{pm} / \mathrm{V}\right.$ in poled fused silica, $\mathrm{d}_{32}=1.7 \mathrm{pm} / \mathrm{V}$ in LBO, $\mathrm{d}_{22}$ $=4.6 \mathrm{pm} / \mathrm{V}$ in $\mathrm{BBO}$ and $\mathrm{d}_{36}=0.8 \mathrm{pm} / \mathrm{V}$ in KDP) that is compensated for by the longer interaction length achievable in optical fibres owing to the low transmission losses over a broad spectral range $(\sim 0.2 \mathrm{~dB} / \mathrm{km})$, and to the $\sim 10$ times lower chromatic dispersion. Additionally, silica fibres exhibits higher damage thresholds $\left(\sim 10^{2} \mathrm{GW} / \mathrm{cm}^{2}\right.$ against $\sim 10^{3} \mathrm{MW} / \mathrm{cm}^{2}$ ) and allow for direct splicing ensuring mechanical stability over time and minimal coupling losses. The years following the first demonstration of all fibre frequency converters have witnessed tremendous technological advances; from the fibre design, to the electrode configurations and finally to the periodic UV erasure enabling extending the length of periodically poled silica fibres (PPSF) over $30 \mathrm{~cm}$ [1]. However, the $\chi^{(2)}$ induced in an optical fibre is not significantly improved. The questions is whether this is because of an intrinsic limit of silica glass and, if that is the case, whether further developments are likely to make PPSF viable devices outside research laboratories.

In the following, state-of-the-art frequency doublers for conversion to the near infrared $(775 \mathrm{~nm})$ and visible $(532 \mathrm{~nm})$ spectral region are presented. Over $230 \mathrm{~mW}$ average second-harmonic at $775 \mathrm{~nm}$ and over $20 \%$ efficiency at $532 \mathrm{~nm}$ are demonstrated in PPSF. Limits and prospects of the current technology are assessed. Finally, high $\chi^{(3)}$ bismuth-zinc-borate ternary systems were studied in effort to increase the $\chi^{(2)}$ susceptibility in glass. It is shown that during poling when the electric field strength in the depletion region builds up above a material dependent threshold the onset of a nonlinear current limits the maximum field to the threshold level. Although these last measurements refer to a glass system different from silica it is anticipated they provide an insight on the factors limiting the $\chi^{(2)}$ in silica.
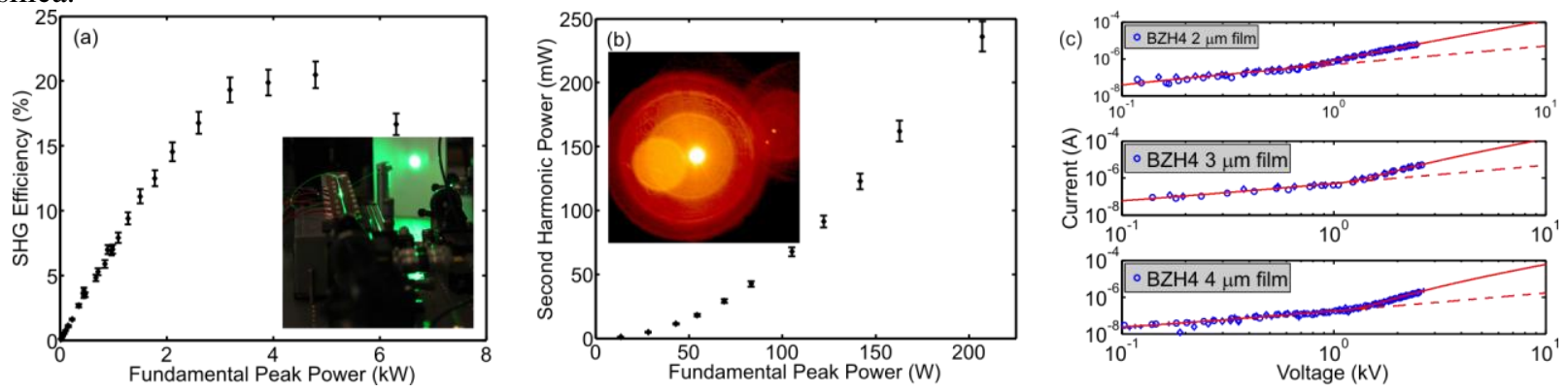

Fig. (a) Plot of the SH average conversion efficiency as a function of the fundamental peak power in a $20 \mathrm{~cm}$ long PPSF. Inset: image of the PPSF emitting green light at $532 \mathrm{~nm}$. (b) Average SH power at $775 \mathrm{~nm}$ measured as a function of fundamental peak power in a $32 \mathrm{~cm}$ long PPSF. Inset - image of the SH light saturating the camera. (c) I-V curves in $\mathrm{Bi}_{2} \mathrm{O}_{3}-\mathrm{ZnO}-\mathrm{B}_{2} \mathrm{O}_{3}$ film (NSG Ltd., $\left[\mathrm{Bi}_{2} \mathrm{O}_{3}\right] \%$ mol $=6.25 \%$ ) on borosilicate

\section{Experiments} substrate showing the onset of nonlinear conductivity.

PPSF were fabricated by UV erasure in uniformly poled germano-silicate twin-hole fibres $\left(\mathrm{d}_{33}=0.051 \pm 0.003\right)$ [1]. Second harmonic (SH) to $532 \mathrm{~nm}$ is obtained by frequency doubling 8 ps pulses emitted by $\mathrm{Nd}: \mathrm{YVO}_{4}$ laser (Super Rapid Lumera Gmbh). Frequency conversion of ultra-short pulses presents a challenge in itself. Earlier attempts to frequency conversion of picosecond or even femtosecond pulses did not exceed $\sim 1 \%$ conversion 
efficiency owing to the constraints imposed by the temporal walk-off between pump and SH pulses. The walk-off length $L_{W-O}=\tau / G V M$, where $G V M$ is the group velocity mismatch and $\tau$ the pulse duration, is calculated as $18.7 \mathrm{~cm}$ for the 8 ps pulses. As the power of the fundamental is increased, the SHG efficiency increases linearly up to peak powers of about $1.5-2 \mathrm{~kW}$ (Fig.1a). The highest external conversion efficiency is recorded as $21 \%$ before it is reduced by competing third-order nonlinear effects. From the SHG measurements the $d_{\text {eff }}$ is estimated in 0.015 $\mathrm{pm} / \mathrm{V}$. The discrepancy with electro-optic measurements reported above is attributed to a non-perfect matching between QPM gratings and laser central wavelength and to the fact that the pulse spectral bandwidth of $0.26 \mathrm{~nm}$ was larger than the acceptance bandwidth of the device. NIR light generation at $770 \mathrm{~nm}$ was demonstrated in an earlier work by frequency doubling $2.5 \mathrm{~ns}$ pulses produced at $3 \mathrm{MHz}$ by a pulsed fibre laser source (custom built by Multitel, Belgium). Over $230 \mathrm{~mW}$ average SH power was produced (Fig.1b). This corresponded to a $15 \%$ efficiency $\left(\eta_{0}=0.717 \% / \mathrm{W} / \mathrm{m}^{2}\right)$ obtained with only $207 \mathrm{~W}$ fundamental peak power (1.6 W average) [2].

\section{Discussion}

The conversion efficiency in PPSF scales according to $\eta=\eta_{0} P_{0} L^{2}$ where $\eta_{0} \propto \mathrm{d}_{\text {eff }}^{2} / A_{\text {ovl }}$ is the normalized conversion efficiency depending solely upon the effective nonlinear coefficient and upon the FH and SH mode overlap. In the NIR conversion experiment the fibre was not optimized for operation at $1550 \mathrm{~nm}$ resulting in a poor mode overlap $\left(A_{o v l}=156 \mu \mathrm{m}^{2}\right)$. A $4-5$ times factor improvement is expected by suitable fibre design. Further gain could be obtained by scaling the length. In the short pulse regime scaling the length is not a viable owing to the insurgence of competing nonlinear effects. It is useful to describe the process in terms of 3 characteristic length scales: 1-The walk-off length, introduced earlier, which represents the length over which good conversion is achieved without significant pulse lengthening. Using $G V M \equiv\left|\left(v_{g}(\lambda / 2)\right)^{-1}-\left(v_{g}(\lambda)\right)^{-1}\right|$ with $\mathrm{v}_{\mathrm{g}}(\lambda)$ being the group velocity at the wavelength $\lambda$ it can be shown that $L_{W-O}=2 \Lambda \mathrm{c} \tau / \lambda$. 2- The length over which the pulse spectrum broadens by a factor of 2 due to self-phase modulation; $L_{n l}=1 / \gamma / P_{0}$ where $\gamma$ is the fibre nonlinear parameter. 3- The QPM-grating length which imposes an acceptance bandwidth for the device that, ideally, accommodates the pulse spectrum $\Delta \lambda ; L_{B W} \approx 0.88 \lambda L / \Delta \lambda$. For transform limited pulses $L_{W-O}=L_{B W}$. The strategy for efficient conversion imposes a device length $L=L_{B W}$ and to limit the pulse peak power to the level for which $L_{n l}=L$ resulting in a useful figure of merit for the frequency doubling of short pulse lasers: $\eta \propto\left(d^{2}{ }_{\text {eff }}\right) \times\left(\Lambda / A_{o v l} / \gamma\right) \times(\lambda / \Delta \lambda)$ where the first factor is solely depending upon the nonlinearity and where the parameters depending on the fibre design and the laser source are lumped in the second and third factor respectively. Both experiments demonstrate already significant conversion efficiency despite the low value of the effective nonlinearity and highlight that Watt-level SHG or single-pass $50 \%$ efficiency in silica optical fibre are definitely feasible.

Nonetheless, increasing the second-order nonlinearity in silica fibres would relax fabrication constraints and most importantly open new avenues for application such as $\mathrm{CW}$ frequency conversion or efficient generation of entangled photon-pairs [3]. According to Kudlinsky's et al. two-charge migration model for the poling of bulk silica glass the maximum stored electric field is reached at the anode surface before hydrogen species $\left(\mathrm{H}^{+}, \mathrm{H}_{3} \mathrm{O}^{+}\right)$are driven into the glass by the high fields [4]. However, this does not necessarily correspond to the highest field strength that silica glass can withstand. In the bismuth zinc borate ternary glass system we tested three films of different thickness deposited on borosilicate substrate [5]. The I-V curves reveal the existence of the onset of a nonohmic current initiating, for all film thicknesses, at field strengths of $2.87 \pm 0.26 \times 10^{8} \mathrm{~V} / \mathrm{m}$ corresponding to $\chi^{(2)}=$ $1.41 \pm 0.12 \mathrm{pm} / \mathrm{V}$ in reasonable agreement with $\chi^{(2)}=1.20 \pm 0.04$ obtained from maker fringe measurements (Fig.1c). The nonlinear current effectively prevents all of the externally applied voltage to drop across the depletion region. While there is no evidence to support that this is happening also in silica glass it is worth noting that a similar mechanism involving e field emission is compatible with the existing models. Mastering the events preceding electrical breakdown in glass may well provide a route towards higher nonlinearity in optical fibres.

\section{References}

[1] A. Canagasabey, M. Ibsen, K.Gallo, A.V. Gladyshev, E.M. Dianov, C. Corbari, P.G. Kazansky, "Aperiodically poled silica fibers for bandwidth control of quasi-phase-matched second-harmonic generation", Optics Letters 35, 724-726 (2010)

[2] A. Canagasabey, C. Corbari, A.V.Gladyshev, F. Liegeois, S. Guillemet, Y. Hernandez, M.V. Yashkov, A. Kosolapov, E.M. Dianov, M. Ibsen, P.G. Kazansky, "High-average-power second-harmonic generation from periodically poled silica fibers", Optics Letters 34, 2483-2485 (2009)

[3] E.Y. Zhu, Z. Tang, L. Qian, L.G. Helt, M. Liscidini, J.E. Sipe, C. Corbari, A. Canagasabey, M. Ibsen, P. G. Kazansky, "Poled-Fiber Source of Polarization-Entangled Photon Pairs", in Specialty Optical Fibers,_OSA Technical Digest (CD), SOWE2 (2011).

[4] A. Kudlinsky, Y. Quiquempois, G. Martinelli, "modeling the $\chi^{(2)}$ susceptibility time evolution in thermally poled fused silica", Optics Express $13,8015-8024(2005)$

[5] C. Corbari, L.C. Ajitdoss, I.C.S. Carvalho, O. Deparis, F.P. Mezzapesa, P.G. Kazansky, K.Sakaguchi, “The problem of achieving high second-order nonlinearities in glasses The role of electronic conductivity in poling of high index glasses", Journal of non Crystalline Solids 356, 2742-2749 (2010) 
